\title{
Evasion-Faced Fast Adaptive Neural Attitude Control for Generic Hypersonic Vehicles with Structural and Parametric Uncertainties
}

\author{
Tian Yan $\mathbb{D}^{1},{ }^{1}$ Yuanli Cai $\mathbb{D}^{1},{ }^{1}$ and Caisheng Wei ${ }^{2}{ }^{2}$ \\ ${ }^{1}$ School of Electronic and Information Engineering, Xian Jiaotong University, Xian 710049, China \\ ${ }^{2}$ School of Aeronautics and Astronautics, Central South University, Changsha 410083, China \\ Correspondence should be addressed to Yuanli Cai; ylicai@mail.xjtu.edu.cn and Caisheng Wei; caisheng_wei@csu.edu.cn
}

Received 14 August 2020; Revised 19 December 2020; Accepted 31 December 2020; Published 27 January 2021

Academic Editor: Libor Pekař

Copyright (c) 2021 Tian Yan et al. This is an open access article distributed under the Creative Commons Attribution License, which permits unrestricted use, distribution, and reproduction in any medium, provided the original work is properly cited.

In general, the evasion task requires the hypersonic vehicles (HSVs) to quickly complete the attitude maneuver in a short time. Moreover, the rapid variation of the flight modes often induces the structural and parametric uncertainties as well as the highly dynamic disturbances of the HSVs. The peculiar and complex characteristics of the evasion process make it difficult to design the evasion-faced flight control systems. In this work, we investigate the fast adaptive control design problem for the generic HSVs under the evasion task. By introducing several especial nonlinear functional vectors and properly designing the adaptive laws, the high dynamic disturbances and uncertainties can be suppressed. To deal with the completed unknown parts of the structural uncertainties and aerodynamic uncertainties caused by evasion maneuver, two radial basis function neural networks (RBFNNs) are introduced as real-time approximators. Furthermore, to improve the response speed of the flight control system, a supertwisting (STW) algorithm-based predictor is used as a feed-forward term of the controller. Consequently, a novel evasion-faced fast adaptive feed-forward control structure has been established for the HSVs. It has been proven that all the signals of the closedloop system are bounded with satisfactory tracking velocity. Finally, the simulation experiment has been set up to show the effectiveness and advantages of the proposed control method.

\section{Introduction}

Hypersonic vehicle has been an active issue for several years due to its splendid maneuverability, strong penetration ability, and fast reaction ability. Compared with traditional ballistic missiles, hypersonic vehicles possess significant advantages such as satisfactory combat effectiveness [1-3]. The main functionality of the hypersonic vehicle control system is to achieve attitude control during flight and complete the tasks transmitted from the guidance subsystem. Because of high flight speed, the accurate information of the dynamic characteristics and atmospheric parameters is difficult to be obtained, which brings difficulties to the design of the flight controller. Moreover, the strong coupling and the complex uncertainties of the HSVs drive the control design difficulties to a higher degree. In summary, the design of the flight controller plays an important role in the development of hypersonic aircraft $[4,5]$.
In recent years, many methods have been applied to the design of hypersonic vehicle controllers, including dynamic inverse method [6], sliding mode control method [7], active disturbance rejection control method [8], back-stepping control, [9] and adaptive control method [4]. Senll et al. proposed a dynamic inverse control method for the nonlinear and strong coupling characteristics of the aircraft during super-maneuvering at a high angle of attack [10]. In [11], a dynamic inverse control method has been proposed for the HSVs, for the purpose of suppressing the intrinsic nonlinear characteristics existing in the HSV system. Li and his colleagues [12] used the dynamic inverse method to achieve complete linearization of the longitudinal model of the HSV s and achieve good control of the hypersonic vehicle's altitude and velocity maneuvers. First order and second order sliding mode control methods are also used in the control design of HSVs [13-15], dealing with the matched uncertainties and improving the robustness of the 
control systems. To achieve the antiwindup tracking control, the technique of terminal sliding mode control has been applied to a class of air-breathing HSVs in [16]. As further development, for the purpose of avoiding the undesired chattering phenomenon, a high-order sliding mode control structure has been designed for the HSVs in [17]. By proposing a sliding mode differentiator, a novel robust back-stepping control scheme has been constructed for the longitudinal dynamics of HSV, eliminating the problem of explosion of terms [18]. In [19-21], the active disturbance rejection control method is used to estimate and compensate the strong uncertainty, channel coupling, and external disturbance of hypersonic vehicles. To solve the actuator nonlinearities, as well as the mismatch between the mathematical model of hypersonic vehicle and the actual dynamic model in complex environment, the adaptive control method is employed in [22, 23]. Existing robust adaptive control methods include [1, 24-26]. The model reference adaptive controller for the HSVs can be found in [27]. In literatures [13, 28-33], several fuzzy adaptive controllers and three adaptive sliding mode controllers of the HSVs have been designed, respectively. Furthermore, adaptive backstepping control structure is proposed in [34, 35]. Faulttolerant adaptive control [36-38] has also been mentioned. In [1], a robust adaptive control law which possesses strong self-adaptability and robustness has been proposed for the HSVs, guaranteeing the stability of the attitude control system. With the aid of the parameter projection technique, a nonlinear robust adaptive control structure has been established for the HSV [25]. In order to adapt to the model change of long-span flight, the model reference adaptive control is used in [27] to make the controlled object track the ideal reference model, so as to obtain the ideal control effect and improve the performance of the control system. In literature [30], fuzzy adaptive method is used to approach the coupling uncertainty of attitude angle to achieve accurate tracking. In [13], a new attitude control algorithm for hypersonic vehicle is proposed by applying fuzzy design to adaptive sliding mode control algorithm. Moreover, in the control of HSVs, the disturbance observers are often used to observe and approximate the disturbances existing in the system, or to compensate the mismatched uncertainties in different channels [11]. It should be pointed out that, in the research on control methods of hypersonic vehicle, various control methods are often used together to solve the control problems of HSVs [6]. In [37], the high-order sliding mode control theory has been combined with the adaptive backstepping control technique, enables the HSV to track speed and height commands stably and effectively, and reduces chattering phenomenon at the same time. In [34], an asymmetric barrier Lyapunov function has been utilized in the adaptive backstepping controller design such that attack angle constraints of the HSV s can be guaranteed during the control process. In [39], a simplified adaptive back-stepping controller using neural networks has been proposed for the longitudinal dynamics of an AHV. Considering the modeling uncertainties and actuator saturation or backlash, an adaptive fault-tolerant controller has been designed in [36]. The state constrained controller of the HSV can be found in $[9,40]$.
However, few control schemes have been investigated for the HSVs under evasion task. In the evasion scenario, the design of the flight control system becomes more challenging because of the complex flight environment, the rapidly changing aerodynamic parameters, and the structural uncertainties. During evasion flight, the drastic change of aerodynamic parameters was a serious challenge to the aircraft control. Because of the peculiar and complex characteristics of the evasion task, a lot of flight controllers are not capable of achieving desired control performance anymore. The control methods mentioned above can hardly satisfy fast tracking requirement of the evasion task. In [41], for a class of uncertain nonlinear systems, a novel sliding mode controller on the basis of LMI has been proposed to satisfy the presence of the sliding mode around the linear surface in the finite time. Furthermore, this method has been extended for the uncertain nonlinear systems with disturbances [42, 43], time-delays, and input nonlinearities, while guaranteeing the robustness and transient performance. In [44], a multivariable super-twisting differentiator has been proposed, which can provide the prediction ability and drastically improve the response speed of the control system. In [45], by using the super-twisting control algorithm, a novel integral sliding mode control strategy has been proposed for the magnetic systems. Compared with the traditional sliding mode control, the symbol function of discontinuous high-frequency switching is replaced by the STW control law such that the output control signal is continuous and the chattering can be essentially eliminated. In [46], a super-twisting observer is designed to estimate the actual value of the brake cylinder, so as to weaken the timevarying disturbance and improve the suppression ability of the system to external disturbance. In [47], an adaptive sliding mode super-twisting differentiator scheme has been proposed, possessing significant estimation and prediction capability. Therefore, it can be known that the STW algorithm can be utilized to construct the feed-forward loop of the controller for the evasion-faced HSVs to enhance the ability of prediction and the response speed. Unfortunately, to the best of the author's knowledge, few control schemes have been proposed for the HSVs under the evasion task, and the evasion-faced fast adaptive flight controller using STW predictor has never been investigated.

Therefore, this paper focuses on the evasion-faced fast adaptive attitude control design problem of the HSVs. Several nonlinear function vectors have been utilized and the high dynamic disturbances and uncertainties can be handled. The RBFNNs are employed as real-time approximators to deal with the completed unknown uncertainties induced by evasion large maneuver. Moreover, an STW algorithmbased predictor is introduced as a feed-forward term to improve the response speed. Compared with the conventional control methods, the proposed control methods possess the following features:

(i) As far as the authors know, it is the first evasionfaced fast adaptive flight controller for the HSVs, suffering from structural and parametric uncertainties and high dynamic disturbances. 
(ii) By using the STW algorithm-based predictor, the response speed of the flight controller can be enhanced and the requirement of the evasion task can be satisfied.

(iii) With the aid of the nonlinear adaptive method and the RBFNNs, the highly dynamic disturbances and unknown uncertainties caused by evasion large maneuver can be effectively suppressed.

\section{Problem Formulation and Preliminaries}

2.1. The Attitude Dynamics of HSV. Based on the results of [2], the attitude dynamics of the HSVs can be described by

$$
\begin{aligned}
& \dot{\eta}=A_{11} \omega+A_{12} C_{F}+\bar{g}+d_{1}(t), \\
& \dot{\omega}=f(\omega)+B C_{M}+d_{2}(t),
\end{aligned}
$$

where $\eta=\left[\alpha, \beta, \gamma_{\nu}\right]^{T}$ denote the vector of the attitude angles, composed of the angle of attack, the sideslip angle, and the bank angle. $\omega=\left[\omega_{x}, \omega_{y}, \omega_{z}\right]^{T}$ is the vector of the attitude angular rates. $C_{F}, C_{M} \in \mathbb{R}\{\hat{3}\}$ represent the coefficients of aerodynamic force and aerodynamic moments, respectively. $d_{1}(t), d_{2}(t) \in \mathbb{R}\{\hat{3}\}$ represent the uncertainties in the attitude angle dynamics and attitude angular velocity dynamics. $A_{11}, A_{12}, B \in \mathbb{R}\{\hat{3} \times 3\}$ are the system matrices, defined as follows:

$$
\begin{aligned}
& A_{11}= {\left[\begin{array}{ccc}
-\tan \beta \cos \alpha & -\tan \beta \sin \alpha & 1 \\
\sin \alpha & -\cos \alpha & 0 \\
\sec \beta \cos \alpha & \sec \beta \sin \alpha & 0
\end{array}\right], } \\
& A_{12}=q S \cdot\left[\begin{array}{ccc}
0 & -\frac{1}{m V \cos \beta} & 0 \\
0 & 0 & \frac{1}{m V} \\
0 \frac{\tan \beta+\tan \theta \sin \gamma_{v}}{m V} & \frac{\tan \theta \cos \gamma_{v}}{m V}
\end{array}\right], \\
& B=q S L \cdot \operatorname{Diag}\left[\frac{1}{I_{x}}, \frac{1}{I_{y}}, \frac{1}{I_{z}}\right],
\end{aligned}
$$

where $I_{x}, I_{y}, I_{z}$ represent the moments of inertia of roll, yaw, and pitch channel; the physical sense of $V, g, m, \theta, q, S$, and $L$ can be founded in [2]. $\bar{g} \in \mathbb{R}\{\hat{3}\}$ is the nonlinear vector caused by the gravitational force; $f(\omega) \in \mathbb{R}\{\hat{3}\}$ is the nonlinear terms related to the inertia parameters. $\bar{g}$ and $f(\omega)$ can be formulated by

$$
\begin{aligned}
\bar{g} & =\left[\frac{g \cos \theta \cos \gamma_{v}}{V \cos \beta}, \frac{g \cos \theta \sin \gamma_{v}}{V},-\frac{g \cos \theta \tan \beta \cos \gamma_{v}}{V}\right]^{T}, \\
f(\omega) & =\left[\frac{I_{y}-I_{z}}{I_{x}} \omega_{y} \omega_{z}, \frac{I_{z}-I_{x}}{I_{y}} \omega_{x} \omega_{z}, \frac{I_{x}-I_{y}}{I_{z}} \omega_{x} \omega_{y}\right]^{T} .
\end{aligned}
$$

The coefficients of aerodynamic force $C_{F}$ can be computed as

$$
C_{F}=C_{F, 0}+C_{F, 1} \eta
$$

The aerodynamic moment coefficients $C_{M}$ can be computed as

$$
C_{M}=C_{M, 0}+C_{M, 1} \eta+C_{M, 2} \omega+C_{M, 3} \delta
$$

where $\delta=\left[\delta_{z}, \delta_{y}, \delta_{x}\right]$ are the reflections of the elevator, rudder, and aileron, representing the vector of control inputs. $C_{F, 0}, C_{M, 0} \in \mathbb{R}\{\hat{3}\}$ are the baseline aerodynamic coefficients. $C_{F, 1}, C_{M, 1}, C_{M, 2}, C_{M, 3} \in \mathbb{R}^{3 \times 3}$ are the aerodynamic partial derivatives.

Accordingly, the aerodynamic uncertainties can be modeled is

$$
\begin{aligned}
C_{F, i} & =\bar{C}_{F, i}+\Delta C_{F, i}, \quad i=0,1, \\
C_{M, i} & =\bar{C}_{M, i}+\Delta C_{M, i}, \quad i=0,1,2,3,
\end{aligned}
$$

where $\bar{C}_{F, i}$ and $\bar{C}_{M, i}$ are known baseline values; $\Delta C_{F, i}, i=0,1$ and $\Delta C_{M, i}, i=0,1,2,3$ denote the aerodynamic perturbations.

In practical, there often exist the modeling errors and structural uncertainties. Let $\Delta A_{11}, \Delta A_{12}, \Delta f(\omega)$, and $\Delta B$ denote the unknown structural uncertainties. Hence, by taking the structural uncertainties and aerodynamic uncertainties into consideration, the following attitude control model can be obtained:

$$
\begin{aligned}
\dot{\eta}= & A_{11} \omega+\Delta A_{11} \omega+A_{12}\left(\bar{C}_{F, 0}+\bar{C}_{F, 1} \eta\right)+\bar{g} \\
& +f_{\eta}(V, \theta, \eta)+d_{1}(t), \\
\dot{\omega}= & f(\omega)+\Delta f(\omega)+B\left[\bar{C}_{M, 0}+\bar{C}_{M, 1} \eta+\bar{C}_{M, 2} \omega\right] \\
& +f_{\omega}(V, \theta, \eta)+\mathscr{B} \delta+\Delta \mathscr{B} \delta+d_{2}(t),
\end{aligned}
$$

where $\mathscr{B}=B \bar{C}_{M, 3}, f_{\eta}(V, \theta, \eta), f_{\omega}(V, \theta, \eta, \omega)$ are the lumped aerodynamic uncertainties, those can be formulated by

$$
\begin{aligned}
f_{\eta}(V, \theta, \eta)= & \Delta A_{12}\left(\bar{C}_{F, 0}+\bar{C}_{F, 1} \eta\right)+\left(A_{12}+\Delta A_{12}\right) \\
& \cdot\left(\Delta C_{F, 0}+\Delta C_{F, 1} \eta\right) \\
f_{\omega}(V, \theta, \eta, \omega)= & \Delta B\left[\bar{C}_{M, 0}+\bar{C}_{M, 1} \eta+\bar{C}_{M, 2} \omega\right] \\
& +(B+\Delta B)\left[\Delta C_{M, 0}+\Delta C_{M, 1} \eta+C_{M, 2} \omega\right]
\end{aligned}
$$

where $\Delta \mathscr{B}$ are the composite uncertainties caused by modeling errors and aerodynamic perturbations, which can be described by

$$
\Delta \mathscr{B}=B \Delta C_{M, 3}+\Delta B\left[\bar{C}_{M, 3}+\Delta C_{M, 3}\right] .
$$

The control objective can be given as follows.

Problem 1. Given the HSV system (7) under the evasion task, it is required to propose a dynamic control law $\delta(t)$ to force the angle of attack $\alpha$, the sideslip angle $\beta$, and the bank angle $\gamma_{V}$ to track the evasion guidance command signals. 


\subsection{Assumptions and Preliminaries}

Assumption 1. It is supposed that the matrices $A_{11}$ and $\mathscr{B}$ are invertible.

Assumption 2. There exist positive constants $\bar{\Delta}_{F}, \bar{\Delta}_{M}$ such that the aerodynamic uncertainties satisfy $\forall t \geq 0,\left\|d_{1}(t)\right\| \leq \bar{\Delta}_{F},\left\|d_{2}(t)\right\| \leq \bar{\Delta}_{M}$.

Assumption 3. In the vicinity of the equilibrium, the uncertain part of $A_{11}$ and $\mathscr{B}$ remains unchanged and cannot change the positive or negative characteristics of $A_{11}$ and $\mathscr{B}$, respectively. In other words, it is assumed that $\left(\mathrm{d} \Delta A_{11} / \mathrm{d} t\right) \approx$ $0,(\mathrm{~d} \Delta \mathscr{B} / \mathrm{d} t) \approx 0, \Lambda_{A}=\Lambda_{A}^{T}, \Lambda_{\mathscr{B}}=\Lambda_{\mathscr{B}}^{T}, \lambda_{\min } \quad\left(\Lambda_{A}\right)>0 \quad$ and $\lambda_{\min }\left(\Lambda_{\mathscr{B}}\right)>0$. Notice that it is defined that $\Lambda_{A}=\left(\left[A_{11}+\right.\right.$ $\left.\left.\Delta A_{11}\right] / A_{11}\right)$ and $\Lambda_{\mathscr{B}}=([\mathscr{B}+\Delta \mathscr{B}] / \mathscr{B})$.

Lemma 1 (see [3]). Given any $\varepsilon>0$ and $z \in \mathbb{R}$, it can be known that

$$
0 \leq\|z\|-\frac{z^{T} z}{\sqrt{z^{T} z+\varepsilon^{2}}}<\varepsilon .
$$

Lemma 2 (see[4]). Consider any time-varying variable $\sigma, \varsigma \in \mathbb{R}^{p}$ satisfying that

$$
\begin{aligned}
& \dot{\sigma}=-k_{11}^{o} \frac{\sigma}{\|\sigma\|}-k_{12}^{o} \sigma+\varsigma, \\
& \dot{\zeta}=-k_{21}^{o} \frac{\sigma}{\|\sigma\|}-k_{21}^{o}+\phi(t),
\end{aligned}
$$

where $\phi(t)$ is a bounded vector, then there exist positive constants $k_{11}^{o}, k_{12}^{o}, k_{21}^{o}, k_{22}^{o}$ such that $\sigma$ and $\varsigma$ can be forced to zero in finite time and remain zero for all subsequent time.

Based on the results provided by $[5,6]$, it can be known that the RBFNNs can approximate the unknown nonlinearities existing in the dynamical systems. As is well known, for any $z \in \Omega$ and constant $\epsilon>0$, given any continuous function $h(z): \Omega \longrightarrow \mathbb{R}$, we can find a $\operatorname{RBFNN} \Theta^{T} \Phi(z)$, satisfying $\sup _{z \in \Omega}\left|h(z)-\Theta^{T} \Phi(z)\right|<\epsilon$. In the RBFNN, $\Theta \in \mathbb{R}^{l}$ denotes the weight vector, $l$ represents the amount of nodes, $\Phi(z)=\left[\phi_{1}(z), \ldots, \phi_{l}(z)\right]^{T}$, and $\phi_{i}(z)=\exp \left(\left(-\left\|z-v_{i}\right\|^{2} /\right.\right.$ $\left.\left.b_{i}^{2}\right)\right), i=1, \ldots, l, b_{i}$ denotes the width of the sigmoid function, and $v_{i}=\left[v_{i, 1}, \ldots, v_{t, n}\right]^{T}$ is the center parameter. Define $\Theta *$ as the optimal weight vector; denote $\varepsilon^{*}$ as the optimal approximation error. Therefore, we know that

$$
h(z)=\left(\Theta^{*}\right)^{T} \Phi(z)+\varepsilon^{*} .
$$

In the following text, the RBFNNs will be utilized to approximate and compensate the unknown nonlinearities existing in the hypersonic dynamics.

\section{Main Results}

3.1. Inner Loop Fast Adaptive NN Controller Design. Let $\alpha_{d}, \beta_{d}, \gamma_{v, d}$ represent the desired trajectories of $\alpha, \beta, \gamma_{v}$. Define $\eta_{d}=\left[\alpha_{d}\right]$. First of all, we can design the following auxiliary system:

$$
\begin{gathered}
\dot{\eta}_{\sigma}=-k_{11}^{o} \frac{\eta_{\sigma}-\eta_{d}}{\left\|\eta_{\sigma}-\eta_{d}\right\|^{(1 / 2)}}-k_{12}^{o}\left(\eta_{\sigma}-\eta_{d}\right)+\varsigma, \\
\dot{\varsigma}=-k_{21}^{o} \frac{\eta_{\sigma}-\eta_{d}}{\left\|\eta_{\sigma}-\eta_{d}\right\|}-k_{22}^{o}\left(\eta_{\sigma}-\eta_{d}\right),
\end{gathered}
$$

where $e_{\sigma d}=\eta_{\sigma}-\eta_{d}, e_{\varsigma}=\varsigma-\dot{\eta}_{d}$; it can be easily obtained that

$$
\begin{gathered}
\dot{e}_{\sigma d}=-k_{11}^{o} \frac{e_{\sigma d}}{\left\|e_{\sigma d}\right\|^{(1 / 2)}}-k_{12}^{o} e_{\sigma d}+e_{\varsigma} \\
\dot{e}_{\varsigma}=-k_{21}^{o} \frac{e_{\sigma d}}{\left\|e_{\sigma d}\right\|}-k_{22}^{o} e_{\sigma d}-\ddot{\eta}_{d} .
\end{gathered}
$$

Then, according to Lemma 2, it can be known that by properly adjusting $k_{11}^{o}, k_{12}^{o}, k_{21}^{o}, k_{22}^{o}, e_{\zeta}$ can be forced to zero in finite time and remain zero for all subsequent time. In other words, $\varsigma$ converges to $\dot{\eta}_{d}$ in finite time and remains here, and besides, there exists a constant $\epsilon_{\varsigma}$ such that $\forall t \geq 0,\left\|e_{\varsigma}\right\| \leq \varepsilon_{\varsigma}$.

Accordingly, we introduce the following auxiliary signal:

$$
\eta_{p}=T_{p} \varsigma+\eta_{d}
$$

where $T_{p}>0$ is a design constant. In the control law, $\eta_{p}$ acts as the command signal, instead of $\eta_{d}$.

Let system output $\eta$ track the predicted desired signal $\eta_{p}$. Define the tracking error as $e_{\eta}=\eta-\eta_{p}$. Define $e_{\eta, d}=\eta-\eta_{d}, e_{\eta, p}=\eta_{p}-\eta_{d}$. It is obvious that $e_{\eta, d}=e_{\eta, p}+e_{\eta}$. By using (15), it can be known that $\dot{\eta}_{p}=T_{p} \dot{\zeta}+\varsigma-e_{\varsigma}$.

In view of the attitude dynamic model (7), the dynamic equation of inner loop tracking error can be written as

$$
\begin{aligned}
\dot{e}_{\eta}= & A_{11} \Lambda_{A} \omega+A_{12}\left(\bar{C}_{F, 0}+\bar{C}_{F, 1} \eta\right)+\bar{g}+f_{\eta}(V, \theta, \eta) \\
& +d_{1}(t)-T_{p} \dot{\varsigma}-\varsigma+e_{\varsigma} .
\end{aligned}
$$

In this subsection, an adaptive controller is designed to force $e_{\eta}$ to converge to a small vicinity of zero. To approximate the unknown aerodynamic uncertainties $f_{\eta}(V, \theta, \eta)$, we introduce a $\operatorname{RBFNN} \Theta_{\eta}^{T} \Phi_{\eta}(V, \theta, \eta)$. Hence, it can be obtained that $f_{\eta}(V, \theta, \eta)=\Theta_{\eta}^{T} \Phi_{\eta}(V, \theta, \eta)+\varepsilon_{\Phi, \eta}$, where $\varepsilon_{\Phi, \eta}$ is the bounded approximation error. Define $\xi_{\eta}=\sup _{t \geq 0}\left\|\varepsilon_{\Phi, \eta}+\varepsilon_{\zeta}+d_{1}(t)\right\|$. Let $\widehat{\Theta}_{\eta}, \widehat{\xi}_{\eta}$ be the estimations of $\Theta_{\eta}, \xi_{\eta}$.

Considering the inner loop tracking error dynamics (16), the indirect virtual control signal is designed as

$$
\omega_{c}=A_{11}^{-1}\left[\begin{array}{c}
-k_{1} e_{\eta}-A_{12}\left(\bar{C}_{F, 0}+\bar{C}_{F, 1} \eta\right)-\widehat{\xi}_{\eta} \varphi_{\eta}\left(e_{\eta}\right) \\
-\widehat{\Theta}_{\eta}^{T} \Phi_{\eta}(V, \theta, \eta)-\bar{g}+T_{p} \dot{\varsigma}+\varsigma
\end{array}\right],
$$

where $k_{1}>0$ is the control gain of the inner loop, $\varphi_{\eta}\left(e_{\eta}\right)=\left(e_{\eta} / \sqrt{e_{\eta}^{T} e_{\eta}+\varepsilon_{\xi, \eta}^{2}}\right) \in \mathbb{R}\{\hat{3}\}$, and $\varepsilon_{\xi, \eta}>0$ is a design constant.

It is easy to know that only using $\omega_{c}$ cannot achieve the inner loop stability, and the induced input uncertainties have to be handled. Hence, we define the final virtual control signal of the inner loop as $\omega_{v}$, which is designed as 


$$
\omega_{v}=\omega_{c}+\omega_{a}
$$

where $\omega_{a}$ is an adaptive compensation term of the input uncertainties, designed as

$$
\omega_{a}=-\widehat{W}_{A} \omega_{c},
$$

where $\widehat{W}_{A}$ is the estimate of $W_{A}, W_{A}=\left(\left[\Lambda_{A}-I\right] / \Lambda_{A}\right)$. By substituting (17) and (18) into (16), we can get that

$$
\begin{aligned}
\dot{e}_{\eta}= & A_{11} \Lambda_{A} e_{\omega}+A_{11}\left[\Lambda_{A}-I\right] \omega_{c}+A_{11} \Lambda_{A} \omega_{a}-k_{1} e_{\eta} \\
& -\widetilde{\Theta}_{\eta}^{T} \Phi_{\eta}(V, \theta, \eta)+\varepsilon_{\Phi, \eta}+d_{1}+e_{\varsigma}-\widehat{\xi}_{\eta} \varphi_{\eta}\left(e_{\eta}\right),
\end{aligned}
$$

where $e_{\omega}=\omega-\omega_{v}$. The adaptive laws of $\widehat{\Theta}_{\eta}, \widehat{W}_{A}$, and $\widehat{\xi}_{\eta}^{T}$ will be obtained in the following text.

Consider the following Lyapunov function:

$$
\begin{aligned}
V_{\eta}= & \frac{1}{2} e_{\eta, p}^{T} e_{\eta, p}+\frac{1}{2} e_{\eta}^{T} e_{\eta}+\frac{1}{2 \Gamma_{\Theta_{\eta}}} \operatorname{Tr}\left(\widetilde{\Theta}_{\eta}^{T} \widetilde{\Theta}_{\eta}\right) \\
& +\frac{1}{2 \Gamma_{W_{A}}} \operatorname{Tr}\left[\widetilde{W}_{A}^{T} \Lambda_{A} \widetilde{W}_{A}\right]+\frac{1}{2 \Gamma_{\xi_{\eta}}} \widetilde{\xi}_{\eta}^{2},
\end{aligned}
$$

where $\widetilde{\Theta}_{\eta}=\widehat{\Theta}_{\eta}-\Theta_{\eta}, \widetilde{W}_{A}=\widehat{W}_{A}-W_{A}, \widetilde{\xi}_{\eta}=\widehat{\xi}_{\eta}-\xi_{\eta} \cdot \Gamma_{\Theta_{\eta}} \Gamma_{W_{A}}$, $\Gamma_{\xi_{\eta}}>0$ are the adaptive gains, and $\operatorname{Tr}(A)$ denotes the trace of matrices $A$. Along (20), we can take the differential of $V_{\eta}$ as

$$
\begin{aligned}
\dot{V}_{\eta}= & -\frac{e_{\eta, p}^{T} e_{\eta, p}}{T_{p}}+e_{\eta, p}^{T} \varepsilon_{p}+e_{\eta}^{T} A_{11} \Lambda_{A} e_{\omega}+e_{\eta}^{T} A_{11}\left[\Lambda_{A}-I\right] \omega_{c} \\
& +e_{\eta}^{T} A_{11} \Lambda_{A} \omega_{a}-k_{1} e_{\eta}^{T} e_{\eta}-e_{\eta}^{T} \widetilde{\Theta}_{\eta}^{T} \Phi_{\eta}(V, \theta, \eta) \\
& +e_{\eta}^{T}\left[\varepsilon_{\Phi, \eta}+d_{1}+e_{\varsigma}\right]-\widehat{\xi}_{\eta} e_{\eta}^{T} \varphi_{\eta}\left(e_{\eta}\right) \\
& +\frac{1}{\Gamma_{\Theta_{\eta}}} \operatorname{Tr}\left(\widetilde{\Theta}_{\eta}^{T} \dot{\widehat{\Theta}}_{\eta}\right)+\frac{1}{\Gamma_{W_{A}}} \operatorname{tr}\left[\widetilde{W}_{A}^{T} \Lambda_{A} \dot{\hat{W}}_{A}\right]+\frac{1}{\Gamma_{\xi}} \widetilde{\xi}_{\eta} \dot{\vec{\xi}}_{\eta},
\end{aligned}
$$

where $\varepsilon_{p}=T_{p} \dot{\varsigma}+\varsigma$. By using Lemma 1, we can get that

$$
e_{\eta}^{T}\left[\varepsilon_{\Phi, \eta}+d_{1}+e_{\varsigma}\right] \leq\left\|e_{\eta}\right\| \xi_{\eta} \leq \xi_{\eta} e_{\eta}^{T} \varphi_{\eta}\left(e_{\eta}\right)+\xi_{\eta} \varepsilon_{\xi, \eta} .
$$

With the aid of (19) and (23), we can rewrite (22) as

$$
\begin{aligned}
\dot{V}_{\eta} \leq- & \frac{e_{\eta, p}^{T} e_{\eta, p}}{T_{p}}+e_{\eta, p}^{T} \varepsilon_{p}+e_{\eta}^{T} A_{11} \Lambda_{A} e_{\omega}-e_{\eta}^{T} A_{11} \Lambda_{A} \widetilde{W}_{a} \omega_{c} \\
& -k_{1} e_{\eta}^{T} e_{\eta}-e_{\eta}^{T} \widetilde{\Theta}_{\eta}^{T} \Phi_{\eta}(V, \theta, \eta) \\
& -\widetilde{\xi}_{\eta} e_{\eta}^{T} \varphi_{\eta}\left(e_{\eta}\right)+\xi_{\eta} \varepsilon_{\xi, \eta} \\
& +\frac{1}{\Gamma_{\Theta_{\eta}}} \operatorname{Tr}\left(\widetilde{\Theta}_{\eta}^{T} \dot{\widehat{\Theta}}_{\eta}\right)+\frac{1}{\Gamma_{W_{A}}} \operatorname{Tr}\left[\widetilde{W}_{A}^{T} \Lambda_{A} \dot{\hat{W}}_{A}\right]+\frac{1}{\Gamma_{\xi_{\eta}}} \widetilde{\xi}_{\eta} \dot{\vec{\xi}}_{\eta} .
\end{aligned}
$$

By using the Young's inequality, it can be known that for any constant $c_{p}>0$, the following inequality holds:

$$
e_{\eta, p}^{T} \varepsilon_{p} \leq c_{p} e_{\eta, p}^{T} e_{\eta, p}+\frac{1}{4 c_{p}} \bar{\varepsilon}_{p}^{T} \bar{\varepsilon}_{p},
$$

where $\bar{\varepsilon}_{p}=\sup _{t \geq 0}\left\|\varepsilon_{p}\right\|$. Hence, we can rewrite (24) as

$$
\begin{aligned}
\dot{V}_{\eta} \leq & -\left(\frac{1}{T_{p}}-c_{p}\right) e_{\eta, p}^{T} e_{\eta, p}+e_{\eta}^{T} A_{11} \Lambda_{A} e_{\omega} \\
& -e_{\eta}^{T} A_{11} \Lambda_{A} \widetilde{W}_{a} \omega_{c}-k_{1} e_{\eta}^{T} e_{\eta}-e_{\eta}^{T} \widetilde{\Theta}_{\eta}^{T} \Phi_{\eta}(V, \theta, \eta) \\
& -\widetilde{\xi}_{\eta} e_{\eta}^{T} \varphi_{\eta}\left(e_{\eta}\right)+\xi_{\eta} \varepsilon_{\xi, \eta}+\frac{1}{4 c_{p}} \bar{\varepsilon}_{p}^{T} \bar{\varepsilon}_{p} \\
& +\frac{1}{\Gamma_{\Theta_{\eta}}} \operatorname{Tr}\left(\widetilde{\Theta}_{\eta}^{T} \dot{\widehat{\Theta}}_{\eta}\right)+\frac{1}{\Gamma_{W_{A}}} \operatorname{Tr}\left[\widetilde{W}_{A}^{T} \Lambda_{A} \dot{\hat{W}}_{A}\right]+\frac{1}{\Gamma_{\xi_{\eta}}} \widetilde{\xi}_{\eta} \dot{\hat{\xi}}_{\eta} .
\end{aligned}
$$

Design the update laws for $\widehat{\Theta}_{\eta}, \widehat{W}_{A}$, and $\widehat{\xi}_{\eta}$ as

$$
\begin{aligned}
\dot{\widehat{\Theta}}_{\eta} & =\Gamma_{\Theta_{\eta}}\left(\Phi_{\eta}(V, \theta, \eta) e_{\eta}^{T}-\sigma_{\Theta_{\eta}} \widehat{\Theta}_{\eta}\right), \\
\dot{\widehat{W}}_{A} & =\Gamma_{W_{A}}\left(\omega_{c} e_{\eta}^{T} A_{11}-\sigma_{W_{A}} \widehat{W}_{A}\right), \\
\dot{\vec{\xi}}_{\eta} & =\Gamma_{\xi_{\eta}}\left(e_{\eta}^{T} \varphi_{\eta}\left(e_{\eta}\right)-\sigma_{\xi_{\eta}} \widehat{\xi}_{\eta}\right),
\end{aligned}
$$

where $\sigma_{\Theta_{\eta}}, \sigma_{W_{A}}, \sigma_{\xi_{\eta}}>0$. Therefore, by substituting the adaptive laws (27) into (26), we can get that

$$
\begin{aligned}
\dot{V}_{\eta} \leq & -\left(\frac{1}{T_{p}}-c_{p}\right) e_{\eta, p}^{T} e_{\eta, p}+e_{\eta}^{T} A_{11} \Lambda_{A} e_{\omega} \\
& -k_{1} e_{\eta}^{T} e_{\eta}+\xi_{\eta} \varepsilon_{\xi, \eta}+\frac{1}{4 c_{p}} \bar{\varepsilon}_{p}^{T} \bar{\varepsilon}_{p} \\
& -\sigma_{\Theta_{\eta}} \operatorname{Tr}\left(\widetilde{\Theta}_{\eta}^{T} \widehat{\Theta}_{\eta}\right)-\sigma_{W_{A}} \operatorname{Tr}\left[\widetilde{W}_{A}^{T} \Lambda_{A} \widehat{W}_{A}\right]-\sigma_{\xi} \widetilde{\xi}_{\eta} \widehat{\xi}_{\eta} .
\end{aligned}
$$

Since

$$
\begin{aligned}
-\operatorname{Tr}\left(\widetilde{\Theta}_{\eta}^{T} \widehat{\Theta}_{\eta}\right) & \leq-\frac{1}{2} \operatorname{Tr}\left(\widetilde{\Theta}_{\eta}^{T} \widetilde{\Theta}_{\eta}\right)+\frac{1}{2} \operatorname{Tr}\left(\Theta_{\eta}^{T} \Theta_{\eta}\right) \\
-\operatorname{Tr}\left[\widetilde{W}_{A}^{T} \Lambda_{A} \widehat{W}_{A}\right] & \leq-\frac{1}{2} \operatorname{Tr}\left[\widetilde{W}_{A}^{T} \Lambda_{A} \widetilde{W}_{A}\right]+\frac{1}{2} \operatorname{Tr}\left[W_{A}^{T} \Lambda_{A} W_{A}\right] \\
-\widetilde{\xi}_{\eta} \widehat{\xi}_{\eta} & \leq-\frac{1}{2} \widetilde{\xi}_{\eta}^{2}+\frac{1}{2} \xi_{\eta}^{2},
\end{aligned}
$$

we can finally get that

$$
\begin{aligned}
\dot{V}_{\eta} \leq & -\left(\frac{1}{T_{p}}-c_{p}\right) e_{\eta, p}^{T} e_{\eta, p}-k_{1} e_{\eta}^{T} e_{\eta}+e_{\eta}^{T} A_{11} \Lambda_{A} e_{\omega} \\
& -\frac{\sigma_{\Theta_{\eta}}}{2} \operatorname{Tr}\left(\widetilde{\Theta}_{\eta}^{T} \widetilde{\Theta}_{\eta}\right)-\frac{\sigma_{W_{A}}}{2} \operatorname{Tr}\left[\widetilde{W}_{A}^{T} \Lambda_{A} \widetilde{W}_{A}\right]-\frac{\sigma_{\xi_{\eta}}}{2} \widetilde{\xi}_{\eta}^{2}+\varepsilon_{\eta},
\end{aligned}
$$

where 


$$
\begin{aligned}
\varepsilon_{\eta}= & \frac{\sigma_{\Theta_{\eta}}}{2} \operatorname{Tr}\left(\Theta_{\eta}^{T} \Theta_{\eta}\right)+\frac{\sigma_{W_{A}}}{2} \operatorname{Tr}\left[W_{A}^{T} \Lambda_{A} W_{A}\right] \\
& +\frac{\sigma_{\xi_{\eta}}}{2} \xi_{\eta}^{2}+\xi_{\eta} \varepsilon_{\xi, \eta}+\frac{1}{4 c_{p}} \bar{\varepsilon}_{p}^{T} \bar{\varepsilon}_{p} .
\end{aligned}
$$

Define $\vartheta=\sup _{t \geq 0}\left\|A_{11} \Lambda_{A}\right\|^{2}$. Then, it is easy to know that for any positive constant $c$, the following inequality holds:

$$
e_{\eta}^{T} A_{11} \Lambda_{A} e_{\omega} \leq \frac{1}{4 c} e_{\eta}^{T} e_{\eta}+c \vartheta e_{\omega}^{T} e_{\omega}
$$

Hence, it follows from (30) that

$$
\begin{aligned}
\dot{V}_{\eta} \leq & -\left(\frac{1}{T_{p}}-c_{p}\right) e_{\eta, p}^{T} e_{\eta, p}-\left(k_{1}-\frac{1}{4 c}\right) e_{\eta}^{T} e_{\eta}+c \vartheta e_{\omega}^{T} e_{\omega} \\
& -\frac{\sigma_{\Theta_{\eta}}}{2} \operatorname{Tr}\left(\widetilde{\Theta}_{\eta}^{T} \widetilde{\Theta}_{\eta}\right)-\frac{\sigma_{W_{A}}}{2} \operatorname{Tr}\left[\widetilde{W}_{A}^{T} \Lambda_{A} \widetilde{W}_{A}\right]-\frac{\sigma_{\xi_{\eta}} \widetilde{\xi}_{\eta}^{2}+\varepsilon_{\eta} .}{2}
\end{aligned}
$$

3.2. Outer Loop Adaptive NN Controller Design. With the aid of second equation of (7), it can be obtained that

$$
\begin{aligned}
\dot{e}_{\omega}= & f(\omega)+\Delta f(\omega)+B\left[\bar{C}_{M, 0}+\bar{C}_{M, 1} \eta+\bar{C}_{M, 2} \omega\right] \\
& +f_{\omega}(V, \theta, \eta)+\mathscr{B} \delta+\Delta \mathscr{B} \delta+d_{2}(t)-\dot{\omega}_{v} .
\end{aligned}
$$

In this section, we design a robust adaptive control structure to ensure closed-loop stability of (34). Identical to the former subsection, we introduce a RBFNN such that $\Delta f(\omega)+f_{\omega}(V, \theta, \eta)=\Theta_{\omega}^{T} \Phi_{\omega}(V, \theta, \eta)+\varepsilon_{\Phi, \omega}$, where $\epsilon_{\Phi, \omega}$ is the bounded approximation error. Let $\xi_{\omega}=\sup _{t \geq 0}\left\|\varepsilon_{\Phi, \omega}+d_{2}(t)\right\|$. Let $\widehat{\Theta}_{\omega}, \widehat{\xi}_{\omega}, \widehat{\vartheta}$ be the estimations of $\Theta_{\omega}, \xi_{\omega}, \vartheta$. Several adaptive laws will be designed to update the parameters $\widehat{\Theta}_{\omega}, \widehat{\xi}_{\omega}, \widehat{\vartheta}$.

Hence, the indirect control signal can be given by

$$
\delta_{c}=\mathscr{B}^{-1}\left[\begin{array}{c}
-k_{2} e_{\omega}-c \widehat{\vartheta} e_{\omega}-f(\omega)-B\left[\bar{C}_{M, 0}+\bar{C}_{M, 1} \eta+\bar{C}_{M, 2} \omega\right] \\
-\widehat{\Theta}_{\omega}^{T} \Phi_{\omega}(V, \theta, \eta, \omega)-\widehat{\xi}_{\omega} \varphi_{\omega}\left(e_{\omega}\right)+\dot{\omega}_{v}
\end{array}\right],
$$

where $k_{2}>0, \varphi_{\omega}\left(e_{\omega}\right)=\left(e_{\omega} / \sqrt{e_{\omega}^{T} e_{\omega}+\varepsilon_{\xi, \omega}^{2}}\right), \varepsilon_{\xi, \omega}>0$.

It is easy to know that only using $\delta_{c}$ cannot achieve the inner loop stability, and the induced input uncertainties have to be handled. Hence, we design the final control signal as

$$
\delta=\delta_{c}+\delta_{a}
$$

where $\delta_{a}$ is an adaptive compensation term of the input uncertainties, designed as

$$
\delta_{a}=\left\{-\widehat{W}_{\mathscr{B}}\right\} \delta_{c},
$$

where $\widehat{W}_{\mathscr{B}}$ is the estimate of $W_{\mathscr{B}}, W_{\mathscr{B}}=\left(\left[\Lambda_{\mathscr{B}}-I\right] / \Lambda_{\mathscr{B}}\right)$. By substituting (35) and (36) into (34), we can get that

$$
\begin{aligned}
\dot{e}_{\omega}= & -k_{2} e_{\omega}-c \widehat{\vartheta} e_{\omega}-\widehat{\Theta}_{\omega}^{T} \Phi_{\omega}(V, \theta, \eta, \omega)+\epsilon_{\Phi, \omega} \\
& +\mathscr{B}\left(\Lambda_{\mathscr{B}}-I\right) \delta_{c}+\mathscr{B} \Lambda_{\mathscr{B}} \delta_{a}+d_{2}(t)-\widehat{\xi}_{\omega}^{T} \varphi_{\omega}\left(e_{\omega}\right) .
\end{aligned}
$$

Select the following Lyapunov function,

$$
\begin{aligned}
V_{\omega}= & \frac{1}{2} e_{\omega}^{T} e_{\omega}+\frac{1}{2 \Gamma_{\Theta_{\omega}}} \operatorname{Tr}\left(\widetilde{\Theta}_{\omega}^{T} \widetilde{\Theta}_{\omega}\right)+\frac{1}{2 \Gamma_{W_{\mathscr{B}}}} \operatorname{Tr}\left(\widetilde{W}_{\mathscr{B}}^{T} \Lambda_{\mathscr{B}} \widetilde{W}_{\mathscr{B}}\right) \\
& +\frac{1}{2 \Gamma_{\xi_{\omega}}} \widetilde{\xi}_{\omega}^{2}+\frac{1}{2 \Gamma_{\vartheta}} \widetilde{\theta}^{2}
\end{aligned}
$$

where $\widetilde{\Theta}_{\omega}=\widehat{\Theta}_{\omega}-\Theta_{\omega}, \widetilde{W}_{\mathscr{B}}=\widehat{W}_{\mathscr{B}}-W_{\mathscr{B}}, \widetilde{\xi}_{\omega}=\widehat{\xi}_{\omega}-\xi_{\omega} \cdot \Gamma_{\Theta_{\omega}}$ $\Gamma_{W_{\mathscr{B}}}, \Gamma_{\xi_{\omega}}>0$. Along (38), the derivative of $V_{\omega}$ can be taken as

$$
\begin{aligned}
\dot{V}_{\omega}= & -k_{2} e_{\omega}^{T} e_{\omega}-c \widehat{\vartheta} e_{\omega}^{T} e_{\omega}-e_{\omega}^{T} \widetilde{\Theta}_{\omega}^{T} \Phi_{\omega}(V, \theta, \eta, \omega) \\
& +e_{\omega}^{T} \mathscr{B}\left(\Lambda_{\mathscr{B}}-I\right) \delta_{c}+e_{\omega}^{T} \mathscr{B} \Lambda_{\mathscr{B}} \delta_{a}+e_{\omega}^{T}\left[\varepsilon_{\Phi, \omega}+d_{2}(t)\right] \\
& -\widehat{\xi}_{\omega} e_{\omega}^{T} \varphi_{\omega}\left(e_{\omega}\right)+\frac{1}{\Gamma_{\Theta_{\omega}}} \operatorname{Tr}\left(\widetilde{\Theta}_{\omega}^{T} \dot{\widehat{\Theta}}_{\omega}\right) \\
& +\frac{1}{\Gamma_{W_{\mathscr{B}}}} \operatorname{Tr}\left(\widetilde{W}_{\mathscr{B}}^{T} \Lambda_{\mathscr{B}} \dot{\widehat{W}}_{\mathscr{B}}\right)+\frac{1}{\Gamma_{\xi_{\omega}}} \widetilde{\xi}_{\omega} \dot{\hat{\xi}}_{\omega}+\frac{1}{\Gamma_{\vartheta}} \tilde{\vartheta} \dot{\hat{\vartheta}} .
\end{aligned}
$$

According to Lemma 1, the following inequality can be obtained:

$$
e_{\omega}^{T}\left[\varepsilon_{\Phi, \omega}+d_{2}(t)\right] \leq\left\|e_{\omega}\right\| \xi_{\omega} \leq \xi_{\omega} e_{\omega}^{T} \varphi_{\omega}\left(e_{\omega}\right)+\xi_{\omega} \varepsilon_{\xi, \omega}
$$

Then, from (41), we know that

$$
\begin{aligned}
\dot{V}_{\omega} \leq & -k_{2} e_{\omega}^{T} e_{\omega}-c \widetilde{\vartheta}_{\omega}^{T} e_{\omega}-c \vartheta e_{\omega}^{T} e_{\omega}-e_{\omega}^{T} \widetilde{\Theta}_{\omega}^{T} \Phi_{\omega}(V, \theta, \eta, \omega) \\
& -e_{\omega}^{T} \mathscr{B} \Lambda_{\mathscr{B}} \widetilde{W}_{\mathscr{B}} \delta_{c}-\widetilde{\xi}_{\omega} e_{\omega}^{T} \varphi_{\omega}\left(e_{\omega}\right)+\xi_{\omega} \varepsilon_{\xi, \omega} \\
& +\frac{1}{\Gamma_{\Theta_{\omega}}} \operatorname{Tr}\left(\widetilde{\Theta}_{\omega}^{T} \dot{\widehat{\Theta}}_{\omega}\right)+\frac{1}{\Gamma_{W_{\mathscr{B}}}} \operatorname{Tr}\left(\widetilde{W}_{\mathscr{B}}^{T} \Lambda_{\mathscr{B}} \dot{\widehat{W}}_{\mathscr{B}}\right) \\
& +\frac{1}{\Gamma_{\xi_{\omega}}} \widetilde{\xi}_{\omega} \dot{\hat{\xi}}_{\omega}+\frac{1}{\Gamma_{\vartheta}} \widetilde{\vartheta} \dot{\widehat{\vartheta}} .
\end{aligned}
$$

In view of (42), the update laws for $\widehat{\Theta}_{\omega}, \widehat{W}_{\mathscr{B}}, \widehat{\vartheta}$, and $\widehat{\xi}_{\omega}$ are designed as follows:

$$
\begin{aligned}
\dot{\hat{\Theta}}_{\omega} & =\Gamma_{\Theta_{\omega}}\left(\Phi_{\omega}(V, \theta, \eta, \omega) e_{\omega}^{T}-\sigma_{\Theta_{\omega}} \widehat{\Theta}_{\omega}\right), \\
\dot{\hat{W}}_{\mathscr{B}} & =\Gamma_{W_{\mathscr{B}}}\left(\delta_{c} e_{\omega}^{T} \mathscr{B}-\sigma_{W_{\mathscr{B}}} \widehat{W}_{\mathscr{B}}\right), \\
\dot{\widehat{\xi}}_{\omega} & =\Gamma_{\xi_{\omega}}\left(e_{\omega}^{T} \varphi_{\omega}\left(e_{\omega}\right)-\sigma_{\xi_{\omega}} \widehat{\xi}_{\omega}\right), \\
\dot{\widehat{\vartheta}} & =\Gamma_{\vartheta}\left(c e_{\omega}^{T} e_{\omega}-\sigma_{\vartheta} \widehat{\vartheta}\right),
\end{aligned}
$$

where $\sigma_{\Theta_{\omega}}, \sigma_{W_{\mathscr{S}}}, \sigma_{\xi_{\omega}}, \sigma_{\vartheta}>0$ are the design constants. Then, from (42) and (43), it can be proved that

$$
\begin{aligned}
\dot{V}_{\omega} \leq & -k_{2} e_{\omega}^{T} e_{\omega}-c \vartheta e_{\omega}^{T} e_{\omega}+\xi_{\omega} \varepsilon_{\xi, \omega}-\sigma_{\Theta_{\omega}} \operatorname{Tr}\left(\widetilde{\Theta}_{\omega}^{T} \widehat{\Theta}_{\omega}\right) \\
& -\sigma_{W_{\mathscr{B}}} \operatorname{Tr}\left(\widetilde{W}_{\mathscr{B}}^{T} \Lambda_{\mathscr{B}} \widehat{W}_{\mathscr{B}}\right)-\sigma_{\xi_{\omega}} \widetilde{\xi}_{\omega} \widehat{\xi}_{\omega}-\sigma_{\vartheta} \widetilde{\vartheta} \widehat{\vartheta} .
\end{aligned}
$$

By using the following inequalities, 


$$
\begin{aligned}
-\operatorname{Tr}\left(\widetilde{\Theta}_{\omega}^{T} \widehat{\Theta}_{\omega}\right) \leq & -\frac{1}{2} \operatorname{Tr}\left(\widetilde{\Theta}_{\omega}^{T} \widetilde{\Theta}_{\omega}\right)+\frac{1}{2} \operatorname{Tr}\left(\Theta_{\omega}^{T} \Theta_{\omega}\right) \\
-\operatorname{Tr}\left(\widetilde{W}_{\mathscr{B}}^{T} \Lambda_{\mathscr{B}} \widehat{W}_{\mathscr{B}}\right) \leq & -\frac{1}{2} \operatorname{Tr}\left(\widetilde{W}_{\mathscr{B}}^{T} \Lambda_{\mathscr{B}} \widetilde{W}_{\mathscr{B}}\right) \\
& +\frac{1}{2} \operatorname{Tr}\left(W_{\mathscr{B}}^{T} \Lambda_{\mathscr{B}} W_{\mathscr{B}}\right) \\
-\widetilde{\xi}_{\omega} \widehat{\xi}_{\omega} \leq & -\frac{1}{2} \widetilde{\xi}_{\omega}^{2}+\frac{1}{2} \xi_{\omega}^{2},-\widetilde{\vartheta} \widehat{\vartheta} \leq-\frac{1}{2} \widetilde{\vartheta}^{2}+\frac{1}{2} \vartheta^{2},
\end{aligned}
$$

we can finally get that

$$
\begin{aligned}
\dot{V}_{\omega} \leq & -k_{2} e_{\omega}^{T} e_{\omega}-c \vartheta e_{\omega}^{T} e_{\omega}-\frac{\sigma_{\Theta_{\omega}}}{2} \operatorname{Tr}\left(\widetilde{\Theta}_{\omega}^{T} \widetilde{\Theta}_{\omega}\right) \\
& -\frac{\sigma_{W_{\mathscr{B}}}}{2} \operatorname{Tr}\left(\widetilde{W}_{\mathscr{B}}^{T} \Lambda_{\mathscr{B}} \widetilde{W}_{\mathscr{B}}\right)-\frac{\sigma_{\xi_{\omega}} \widetilde{\xi}_{\omega}^{2}-\frac{\sigma_{\vartheta} \widetilde{\vartheta}^{2}}{2}+\varepsilon_{\omega},}{2}
\end{aligned}
$$

where

$$
\begin{gathered}
\varepsilon_{\omega}=\xi_{\omega} \varepsilon_{\xi, \omega}+\frac{\sigma_{\Theta_{\omega}}}{2} \operatorname{Tr}\left(\Theta_{\omega}^{T} \Theta_{\omega}\right)+\frac{\sigma_{W_{\mathscr{B}}}}{2} \operatorname{Tr} \\
\cdot\left(W_{\mathscr{B}}^{T} \Lambda_{\mathscr{B}} W_{\mathscr{B}}\right)+\frac{\sigma_{\xi_{\omega}}}{2} \xi_{\omega}^{2}+\frac{\sigma_{\vartheta}}{2} \vartheta^{2} .
\end{gathered}
$$

The structure of the proposed evasion-faced fast attitude control method is given by Figure 1 .

\subsection{Stability Analysis}

Theorem 1. Consider the hypersonic attitude dynamics (1). If the virtual control laws are designed as (17)-(19), the actual control laws are designed as (35)-(37), the adaptive laws are selected as (27) and (43), then the closed-loop system consisting of the adaptive controller, the auxiliary system (13), and the parameter update laws (27) and (43) is stable; all the signals and the equilibrium of closed-loop system are bounded, and the angle of attack $\alpha$, the sideslip angle $\beta$, and the bank angle $\gamma_{v}$ will converge to the desired values asymptotically.

Proof. Consider the following Lyapunov function:

$$
V=V_{\eta}+V_{\omega} \text {, }
$$

where $V_{\eta}$ and $V_{\omega}$ have been given in (21) and (39), respectively. Then, according to the analysis provided in Sections 2.1 and 2.2 , we can arrive at that

$$
\begin{aligned}
\dot{V} \leq & -\left(\frac{1}{T_{p}}-c_{p}\right) e_{\eta, p}^{T} e_{\eta, p}-\left(k_{1}-\frac{1}{4 c}\right) e_{\eta}^{T} e_{\eta}-k_{2} e_{\omega}^{T} e_{\omega} \\
& -\frac{\sigma_{\Theta_{\eta}}}{2} \operatorname{Tr}\left(\widetilde{\Theta}_{\eta}^{T} \widetilde{\Theta}_{\eta}\right)-\frac{\sigma_{W_{A}}}{2} \operatorname{Tr}\left[\widetilde{W}_{A}^{T} \Lambda_{A} \widetilde{W}_{A}\right]-\frac{\sigma_{\xi_{\eta}} \widetilde{\xi}_{\eta}^{2}+\varepsilon_{\eta}}{2} \\
& -\frac{\sigma_{\Theta_{\omega}}}{2} \operatorname{Tr}\left(\widetilde{\Theta}_{\omega}^{T} \widetilde{\Theta}_{\omega}\right)-\frac{\sigma_{W_{\mathscr{B}}}}{2} \operatorname{Tr}\left(\widetilde{W}_{\mathscr{B}}^{T} \Lambda_{\mathscr{B}} \widetilde{W}_{\mathscr{B}}\right)-\frac{\sigma_{\xi_{\omega}} \widetilde{\xi}_{\omega}^{2}}{2} \\
& -\frac{\sigma_{\Im}}{2} \widetilde{\vartheta}^{2}+\varepsilon_{\omega} .
\end{aligned}
$$

$$
\dot{V} \leq-\lambda V+\varepsilon,
$$

where

$$
\lambda=\min \left\{\begin{array}{l}
2\left(\frac{1}{T_{p}}-c_{p}\right),\left(2 k_{1}-\frac{1}{2 c}\right), 2 k_{2}, \Gamma_{\Theta_{\eta}} \sigma_{\Theta_{\eta}}, \\
\sigma_{W_{A}} \Gamma_{W_{A}}, \sigma_{\xi_{\eta}} \Gamma_{\xi_{\eta}}, \sigma_{\Theta_{\omega}} \Gamma_{\Theta_{\omega}}, \\
\sigma_{W_{\mathscr{S}}} \Gamma_{W_{\mathscr{S}}}, \sigma_{\xi_{\omega}} \Gamma_{\xi_{\omega}}, \sigma_{\vartheta} \Gamma_{\vartheta}, \\
\varepsilon=\varepsilon_{\eta}+\varepsilon_{\omega} .
\end{array}\right.
$$

Hence, it is easy to know that $\forall t \geq 0,0 \leq V(t) \leq$ $\max \{(\varepsilon /(2 \lambda)), V(0)\}$. As a consequence, the boundness of all the signals in the closed-loop system can be ensured. Then, by considering the definition of $e_{\eta, p}$ and $e_{\eta}$, it can be proved that $\eta$ can converge to a small neighborhood of $\eta_{d}$ asymptotically. In other words, the angle of attack $\alpha$, the sideslip angle $\beta$, and the bank angle $\gamma_{v}$ can track the desired values asymptotically. The proof is completed.

\section{Simulation Study}

In the section, two simulation experiments are set up to show the effectiveness and advantages of the proposed control method. The first simulation considered the HSVs suffering from different uncertainties, and the effectiveness and robustness of the proposed adaptive neural network control method can be revealed. The second simulation involved the proposed fast adaptive NN control (FANNC) method, the adaptive NN control (ANNC) methods without super-twist predictor, and the conventional model reference adaptive control method which are also included for comparison.

In the simulation, the parameters of the hypersonic vehicle are selected as Table 1 . The initial attitude dynamics of the hypersonic vehicle are $\alpha=\beta=\gamma_{v}=0, \omega_{x}=\omega_{y}=$ $\omega_{z}=0$. Considering the evasion requirement, we select the desired signal as

$$
\begin{aligned}
\alpha_{d}(t) & =5 \sin \left(\frac{2 \pi t}{10}\right)+5 \sin \left(\frac{\pi t}{10}\right), \\
\beta_{d}(t) & =0, \\
\gamma_{v, d}(t) & =5 \sin \left(\frac{2 \pi t}{10}\right)+3.6 \cos \left(\frac{\pi t}{10}\right) .
\end{aligned}
$$

The uncertainties of the three cases considered in the simulations are given in Tables 2 and 3 .

The initial values of the adaptive control parameters matrix are $\widehat{\Theta}_{\eta 0}, \widehat{\Theta}_{\omega 0}=0^{9 \times 3}, \widehat{W}_{A 0}, \widehat{W}_{B 0}=0^{3 \times 3}, \eta_{\sigma 0}, \varsigma_{0}=0^{3 \times 1}$, and $\widehat{\xi}_{\eta 0}, \widehat{\xi}_{\omega 0}, \widehat{\vartheta}_{0}=0$. The fixed value parameters of the hypersonic vehicle control system are chosen as $T_{p}=0.01$, $c=1, k_{11}=0.5, k_{12}=3, k_{21}=5, k_{22}=10, k_{1}=12, k_{2}=18$, $\Gamma_{\Theta_{\eta}}=5, \Gamma_{W_{A}}=5, \Gamma_{\Theta_{\omega}}=5, \Gamma_{W_{B}}=5, \Gamma_{\xi_{\eta}}=5, \Gamma_{\xi_{\omega}}=5, \Gamma_{\vartheta}=5$, $\sigma_{\Theta_{\eta}}=3, \sigma_{W_{A}}=3, \sigma_{\Theta_{\omega}}=3, \sigma_{W_{B}}=3, \sigma_{\xi_{\eta}}=3, \sigma_{\xi_{\omega}}=3$, and $\sigma_{9}=3$. The initial auxiliary system states are defined as follows: $\eta_{\sigma 0}=[0,0,0]^{T}$ and $\varsigma_{0}=[0,0,0]^{T}$. 


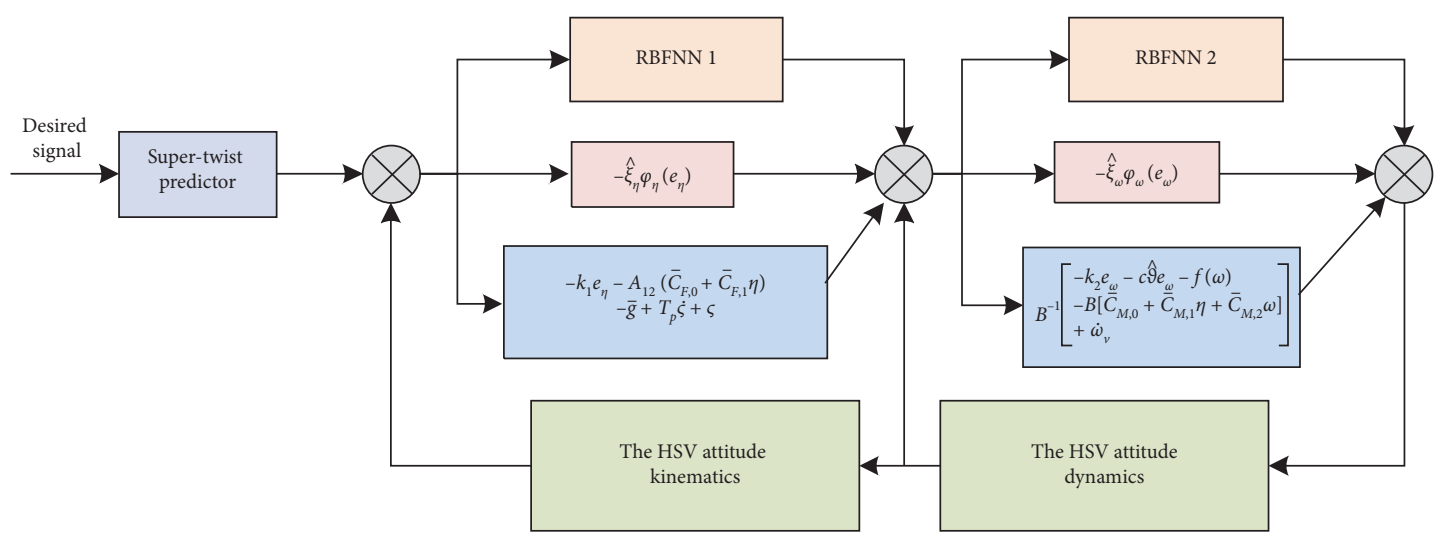

FIGURE 1: The structure of the proposed evasion-faced fast attitude control method.

TABLE 1: The parameters of the hypersonic vehicle.

\begin{tabular}{lccc}
\hline$m$ & $S$ & $L$ & $I_{x x}$ \\
\hline $4900 \mathrm{~kg}$ & $7.565 \mathrm{~m}^{2}$ & $4.55 \mathrm{~m}$ \\
$I_{y y}$ & $I_{z z}$ & $v$ & $2345 \mathrm{~kg} \cdot \mathrm{m}^{2}$ \\
$24706 \mathrm{~kg} \cdot \mathrm{m}^{2}$ & $24309 \mathrm{~kg} \cdot \mathrm{m}^{2}$ & $1860(\mathrm{~m} / \mathrm{s})$ & \\
\hline
\end{tabular}

TABle 2: The uncertainties of the three cases.

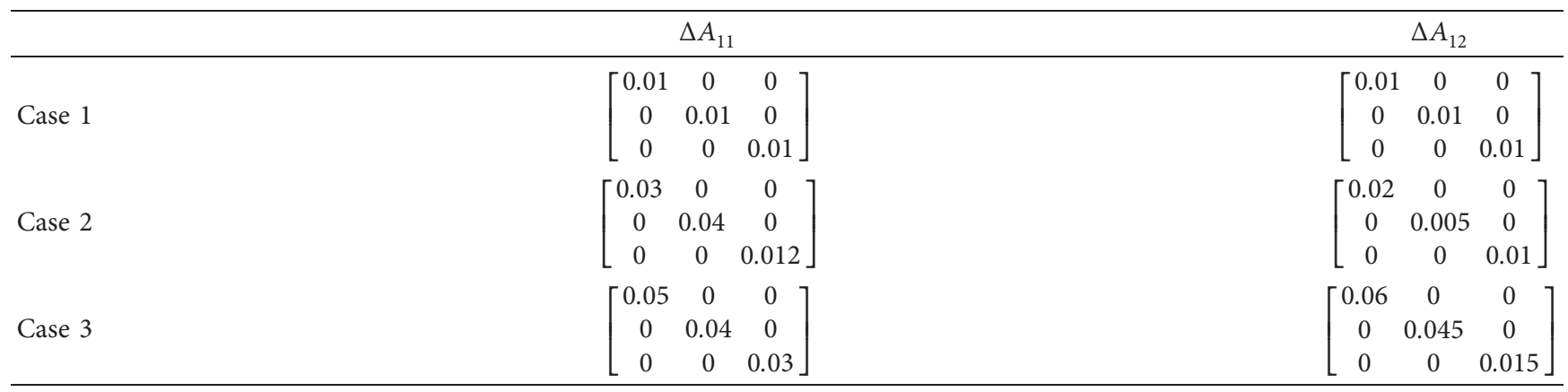

TABLE 3: The uncertainties of the three cases.

\begin{tabular}{|c|c|c|c|c|}
\hline & $\Delta f$ & \multicolumn{3}{|c|}{$\Delta B$} \\
\hline Case 1 & {$\left[\begin{array}{l}0.1 \\
0.1 \\
0.1\end{array}\right]$} & {$\left[\begin{array}{c}0.01 \\
0 \\
0\end{array}\right.$} & $\begin{array}{c}0 \\
0.01 \\
0\end{array}$ & $\left.\begin{array}{c}0 \\
0 \\
0.01\end{array}\right]$ \\
\hline Case 2 & {$\left[\begin{array}{c}0.05 \\
0.2 \\
0.25\end{array}\right]$} & {$\left[\begin{array}{c}0.015 \\
0 \\
0\end{array}\right.$} & $\begin{array}{c}0 \\
0.01 \\
0\end{array}$ & $\begin{array}{c}0 \\
0 \\
0.03\end{array}$ \\
\hline Case 3 & {$\left[\begin{array}{l}0.35 \\
0.24 \\
0.25\end{array}\right]$} & {$\left[\begin{array}{c}0.02 \\
0 \\
0\end{array}\right.$} & $\begin{array}{c}0 \\
0.04 \\
0\end{array}$ & $\begin{array}{c}0 \\
0 \\
0.03\end{array}$ \\
\hline
\end{tabular}

The simulation results are given in Figures 2-6. The tracking results in cases $1-3$ are respectively given in Figures 2-4, from which we can see that the attitude angle of the three channels all track the expected signal quickly and steadily in different cases. The weights and the adaptive parameters are given in Figures 5 and 6. It is obvious that the adaptive parameters are all bounded. The robustness properties of the proposed algorithm can be revealed therefore.

To illustrate the superiority of the proposed adaptive control scheme, we further apply the adaptive NN control methods without using the super-twist predictor and the conventional model reference adaptive control method to 


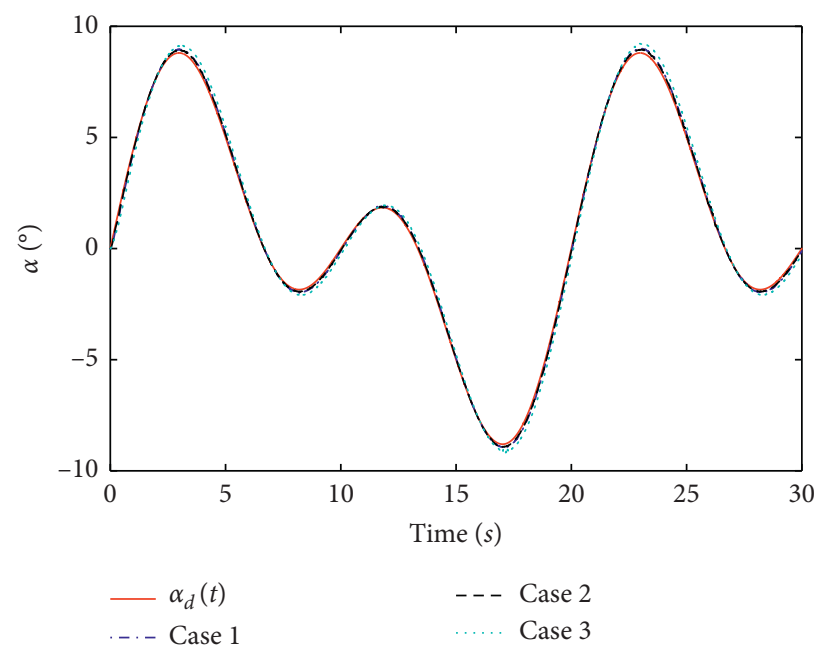

FIGURE 2: The tracking performance of $\alpha_{d}(t)$ under three cases.

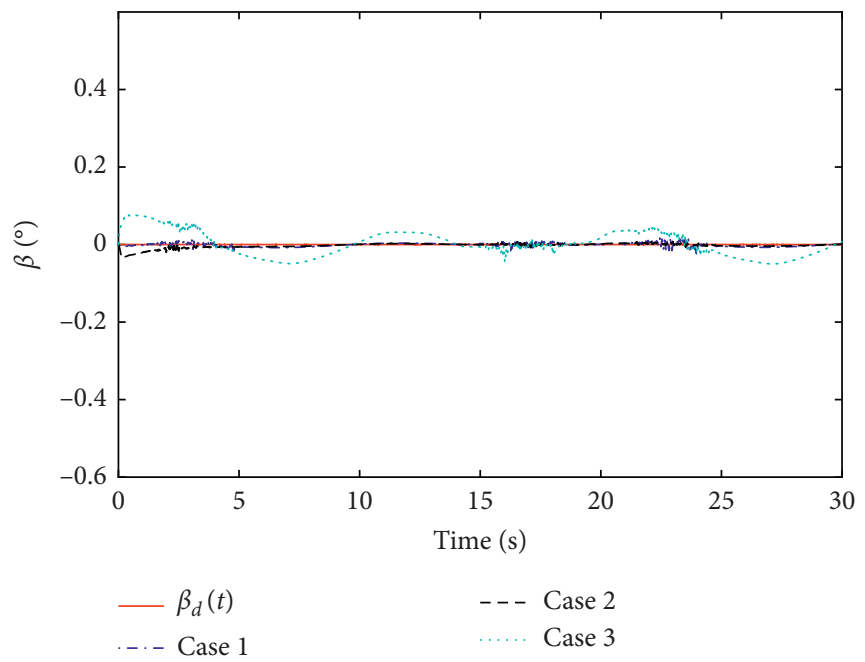

FIgURE 3: The tracking performance of $\beta_{d}(t)$ under three cases.

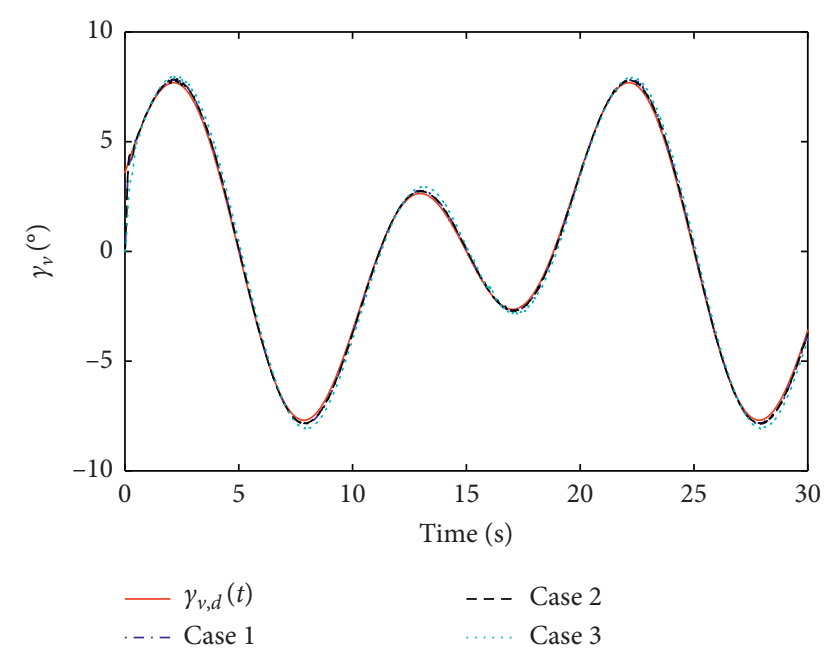

FIGURE 4: The tracking performance of $\gamma_{v, d}(t)$ under three cases.

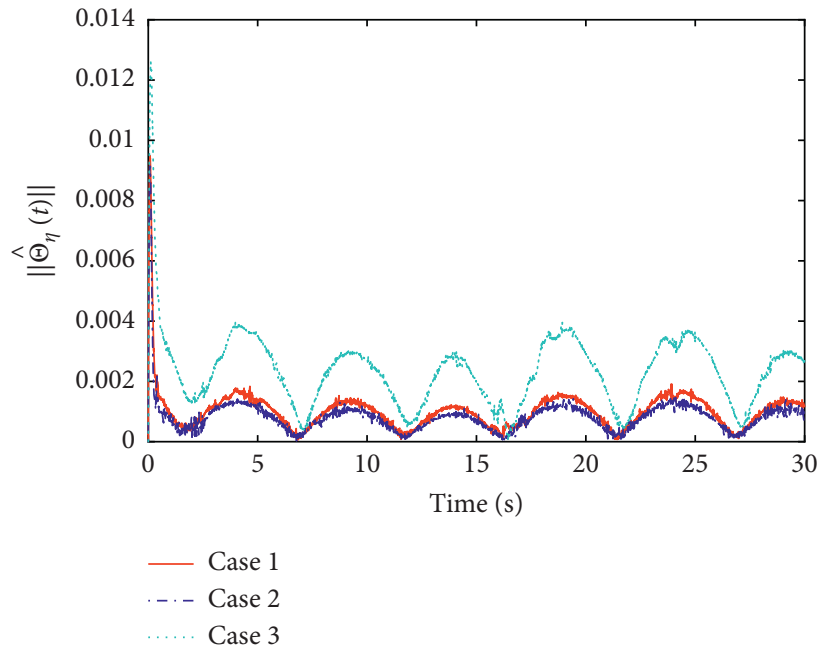

FIgURE 5: The norm of the NN weights matrix under three cases. 


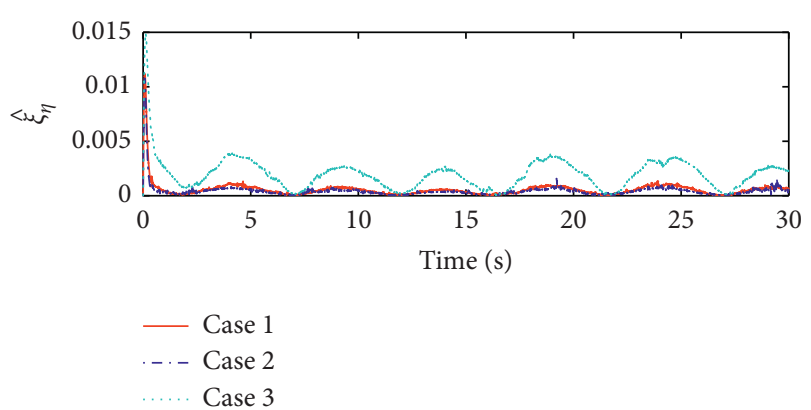

(a)

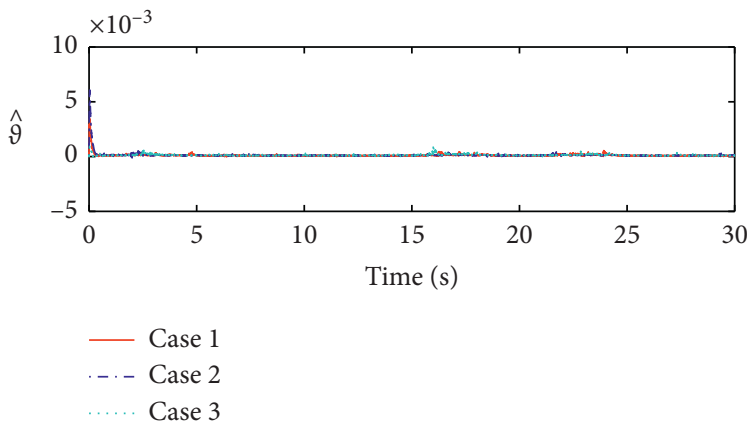

(b)

Figure 6: The adaptive parameters under three cases.

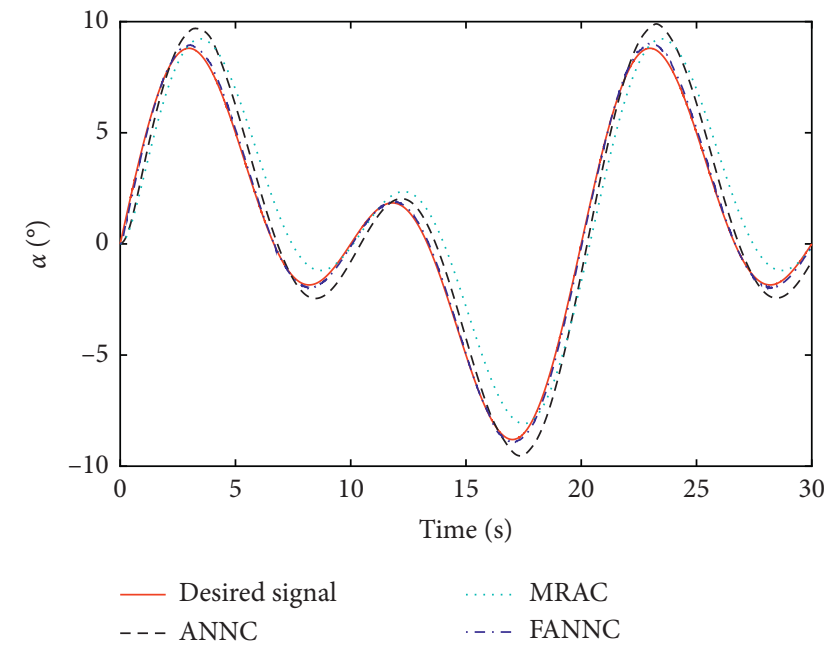

Figure 7: The tracking performance of $\alpha_{d}(t)$ of the three methods.

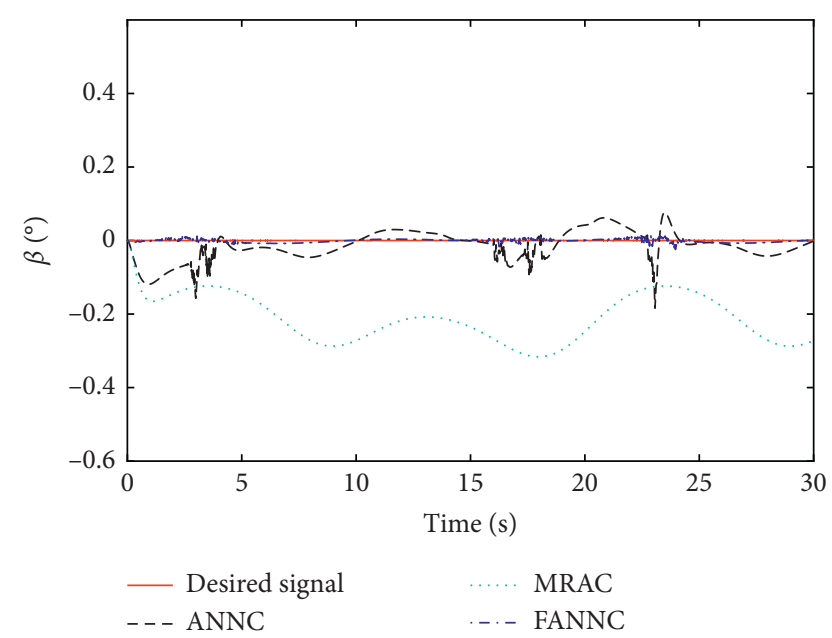

FIGURE 8: The tracking performance of $\beta_{d}(t)$ of the three methods.

the attitude control of the HSVs. Figures 7-9 respectively show the tracking results under three control schemes; Table 4 provides the comparative control performance, from which we can conclude that the tracking error is

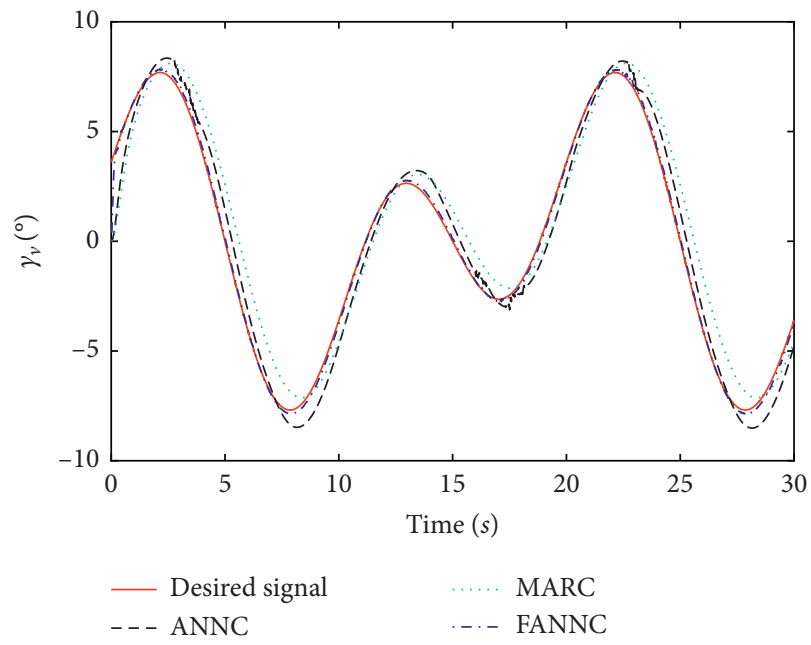

FIGURE 9: The tracking performance of $\gamma_{v, d}(t)$ of the three methods.

TABle 4: The performance comparison of the three control methods.

\begin{tabular}{|c|c|c|c|}
\hline & FANNC & MRAC & ANNC \\
\hline Pitch channel & $\begin{array}{l}\text { Maximal time } \\
\text { delay } 0.01 \\
\text { Maximal } \\
\text { tracking error } \\
0.05\end{array}$ & $\begin{array}{l}\text { Maximal time } \\
\text { delay } 0.5 \\
\text { Maximal } \\
\text { tracking error } \\
0.6\end{array}$ & $\begin{array}{c}\text { Maximal } \\
\text { time delay } 0.35 \\
\text { Maximal } \\
\text { tracking error } \\
0.4\end{array}$ \\
\hline Yaw channel & $\begin{array}{l}\text { Maximal time } \\
\text { delay } 0 \\
\text { Maximal } \\
\text { tracking error } \\
0.005 \\
\end{array}$ & $\begin{array}{l}\text { Maximal time } \\
\text { delay } 0 \\
\text { Maximal } \\
\text { tracking error } \\
0.09\end{array}$ & $\begin{array}{l}\text { Maximal time } \\
\text { delay } 0 \\
\text { Maximal } \\
\text { tracking error } \\
0.36\end{array}$ \\
\hline Roll channel & $\begin{array}{l}\text { Maximal time } \\
\text { delay } 0.02 \\
\text { Maximal } \\
\text { tracking error } \\
0.05\end{array}$ & $\begin{array}{l}\text { Maximal time } \\
\text { delay } 0.4 \\
\text { Maximal } \\
\text { tracking error } \\
0.95\end{array}$ & $\begin{array}{c}\text { Maximal time } \\
\text { delay } 0.45 \\
\text { Maximal } \\
\text { tracking error } \\
0.8\end{array}$ \\
\hline
\end{tabular}

unacceptable without proper adaptive compensation, and the phase lag may be induced if the STW predictor is deprecated. The reason for this difference is that the proposed method introduces adaptive neural networks and the 
STW predictor, which can tolerate the uncertainty in the flight process and improve the tracking velocity. The superiority of the proposed control scheme can be verified therefore.

\section{Conclusions}

In this paper, a novel evasion-faced fast adaptive feed-forward control structure has been synthesized. By using a super-twisting (STW) algorithm-based predictor, the requirement of the response speed of the flight control system can be satisfied. By employing two RBFNNs as real-time approximators, the unknown uncertainties existing in the fight control system can be handled. Moreover, the adaptive laws have been properly designed such that the difficulties caused by the highly dynamic disturbances can be circumvented. As a consequence, the peculiar and complex attitude control problem for the evasion tasks can be addressed. Simulation results show that by using the proposed fast adaptive feed-forward control structure, the tracking error can be forced to converge into a desired domain and the phase lag can be reduced. Our future work includes the evasion-faced integrated guidance and control design for the HSVs and the parametric optimization for the evasion-faced flight control system of the HSVs.

\section{Data Availability}

The data used to support the study are available from the corresponding author upon request.

\section{Conflicts of Interest}

The authors declare that they have no conflicts of interest.

\section{References}

[1] Z. Shi and C. He, "Robust adaptive attitude control for hypersonic reentry vehicle," Electric Machines and Control, vol. 22, no. 7, pp. 118-124, 2018.

[2] Z. Guo, J. Zhou, and J. Guo, "Novel coupling based attitude control system design for hypersonic vehicles," Journal of Astronautics, vol. 38, pp. 270-278, 2017.

[3] H.-Y. Qiao, H. Meng, M.-J. Wang, W. Ke, and J.-G. Sun, "Adaptive control for hypersonic vehicle with input saturation and state constraints," Aerospace Science and Technology, vol. 84, no. 1, pp. 107-119, 2019.

[4] C. Huang, J. Guo, W. Bai, and L. Kunfeng, "Adaptive attitude control for hypersonic vehicle based on sigmoid function," Computer Measurement \& Control, vol. 26, no. 11, pp. 145149, 2018.

[5] J. Wang, H. Liang, X. Wu, and Q. Fu, "Continuous terminal sliding mode attitude control for hypersonic aircrafts," Journal of Harbin Engineering University, vol. 37, no. 2, pp. 187-191, 2016.

[6] P. Wang, Research on attitude control method for hypersonic cruise vehicle, Ph.D. thesis, National University of Defense Technology, Changsha, China, 2013.

[7] H. Sun, S. Li, and C. Sun, "Finite time integral sliding mode control of hypersonic vehicles," Nonlinear Dynamics, vol. 73, no. 1-2, pp. 229-244, 2013.
[8] X. Shao and H. Wang, "Active disturbance rejection based trajectory linearization control for hypersonic reentry vehicle with bounded uncertainties," ISA Transactions, vol. 54, pp. 27-38, 2015.

[9] X. Bu, X. Wu, D. Wei, and J. Huang, "Neural-approximationbased robust adaptive control of flexible air-breathing hypersonic vehicles with parametric uncertainties and control input constraints," Information Sciences, vol. 346-347, pp. 29-43, 2016.

[10] S. A. Snell, D. F. Nns, and W. L. Arrard, "Nonlinear inversion flight control for a supermaneuverable aircraft," Journal of Guidance, Control and Dynamics, vol. 15, no. 4, pp. 976-984, 2015.

[11] X. Liu, Y. Wu, and P. Xu, "Attitude control of hypersonic vehicle considering input saturation," Journal of System Simulation, vol. 31, no. 11, pp. 379-387, 2019.

[12] H. Li, W.-C. Sun, and Z.-Y. Li, "Index approach law based sliding control for a hypersonic aircraft," in Proceedings of the 2nd International Symposium on Systems and Control in Aerospace and Astronautics, pp. 1-5, Shenzhen, China, December 2008.

[13] P. Guan and Z. He, "Adaptive fuzzy sliding mode control for hypersonic vehicle attitude," Control Engineering of China, vol. 25, no. 7, pp. 3-8, 2018.

[14] P. Guan, Z. He, and X. Ge, "Second-order sliding mode attitude control based on fuzzy control for hypersonic vehicle," Control and Decision, vol. 34, no. 9, pp. 1901-1908, 2019.

[15] P. Chu, K. Ni, L. Cheng, and Q. Zhang, "Robust control of hypersonic morphing vehicle based on backstepping sliding mode control," Computer Simulation, vol. 448, no. 8, pp. 8-12, 2018.

[16] H. An, H. Xia, and C. Wang, "Finite-time output tracking control for air-breathing hypersonic vehicles with actuator constraints," in Proceedings of the Institution of Mechanical Engineers Part G Journal of Aerospace Engineering, vol. 231, no. 14, pp. 2578-2593, 2016.

[17] J. Wang, Q. Zong, R. Su, and B. Tian, "Continuous high order sliding mode controller design for a flexible air-breathing hypersonic vehicle," ISA Transactions, vol. 53, no. 3, pp. 690-698, 2014.

[18] X. W. Bu, X. Y. Wu, R. Y. Bai, and Z. Ma, "Sliding-modedifferentiator-based robust backstepping control of airbreathing hypersonic vehicles," Journal of Solid Rocket Technology, vol. 38, no. 1, pp. 12-17, 2015.

[19] M. Piao, Z. Yang, M. Sun, J. Huang, and Z. Chen, "Attitude control synthesis of unstable hypersonic vehicle with aeroservoelastic effect," SCIENTIA SINICA Technologica, vol. 49, pp. 825-839, 2019.

[20] M. Piao, M. Sun, J. Huang, and Z. Chen, "Robust attitude control of hypersonic vehicle based on active disturbance rejection control," Control Engineering of China, vol. 26, no. 9, pp. 25-33, 2019.

[21] H. Du, Y. Fan, and J. Yan, "Active disturbance rejection control for air-breathing hypersonic vehicle," Jisuanji $Y u$ Xiandaihua, vol. 214, no. 6, pp. 1-4, 2013.

[22] W. Yong, "Application of characteristic model based decentralized adaptive attitude control in the hypersonic vehicle," Journal of Astronautics, vol. 33, no. 10, pp. 14131422, 2012.

[23] L. Fiorentini, A. Serrani, M. A. Bolender, and D. B. Doman, "Nonlinear robust/adaptive controller design for an airbreathing hypersonic vehicle model (preprint)," AIAA Journal, vol. 6, no. 15, pp. 2007-6329, 2013. 
[24] T. Zhang, J. Zhou, and J. Guo, "A study on robust adaptive controll method for hypersonic vehicle," Journal of Astronautics, vol. 34, no. 3, pp. 384-388, 2013.

[25] L. Fiorentini, A. Serrani, M. A. Bolender, and D. B. Doman, "Nonlinear robust adaptive control of flexible air-breathing hypersonic vehicles," Journal of Guidance, Control, and Dynamics, vol. 32, no. 2, pp. 402-417, 2009.

[26] X. W. Bu, X. Y. Wu, Z. Ma, and R. Zhang, "State-reconstruction-based robust backstepping control of air-breathing hypersonic vehicles," Journal of Solid Rocket Technology, vol. 38, no. 3, pp. 314-355, 2015.

[27] X. Cheng, L. Liu, and G. Tang, "The research on fuzzy model reference adaptive control for hypersonic vehicle," Aerospace Control, vol. 32, no. 4, pp. 13-18, 2014.

[28] Z. Li, C.-Y. Su, G. Li, and H. Su, "Fuzzy approximation-based adaptive backstepping control of an exoskeleton for human upper limbs," IEEE Transactions on Fuzzy Systems, vol. 23, no. 3, pp. 555-566, 2015.

[29] S. Tong, S. Sui, and Y. Li, "Adaptive fuzzy decentralized tracking fault-tolerant control for stochastic nonlinear largescale systems with unmodeled dynamics," Information Sciences, vol. 289, pp. 225-240, 2014.

[30] C. Hu and Y. Liu, "Fuzzy adaptive attitude control based on eso for hypersonic vehicles," Aerospace Control, vol. 33, no. 3, pp. 47-53, 2015.

[31] Q. Zong, J. Wang, and Y. Tao, “Adaptive high-order dynamic sliding mode control for a flexible air-breathing hypersonic vehicle," International Journal of Robust \& Nonlinear Control, vol. 23, no. 15, 2013.

[32] B. Tian and W. Fan, "Adaptive high order sliding mode controller design for hypersonic vehicle," in Proceedings of the 10th IEEE International Conference on Control \& Automation, Hangzhou, China, June 2013.

[33] H. Li and Y. Cai, "Adaptive high-order sliding mode control design for a hypersonic vehicle," System Simulation Technology \& Application, vol. 16, pp. 339-343, 2015.

[34] C. Dong, Y. Liu, and Q. Wang, "Adaptive backstepping controller design for hypersonic vehicle with limited angle-ofattack," Journal of Astronautics, vol. 41, no. 2, pp. 174-181, 2020.

[35] Y. Feng, Y.-X. Wu, Y.-M. Hu, and Su, “Adaptive backstepping control of a class of uncertain nonlinear systems with PrandtlIshlinskii hysteresis,"vol. 2, pp. 697-701, in Proceedings of the International Conference on Machine Learning and Cybernetics, vol. 2, , IEEE, Guangzhou, China, August 2005.

[36] Q. Shen, B. Jiang, and V. Cocquempot, "Fault-tolerant control for T-S fuzzy systems with application to near-space hypersonic vehicle with actuator faults," IEEE Transactions on Fuzzy Systems, vol. 20, no. 4, pp. 652-665, 2012.

[37] H. An, Q. Wu, C. Wang, and X. Cao, "Simplified fault-tolerant adaptive control of air-breathing hypersonic vehicles," International Journal of Control, vol. 93, no. 8, pp. 1964-1979, 2020.

[38] H. An, J. Liu, C. Wang, and L. Wu, "Approximate backstepping fault-tolerant control of the flexible air-breathing hypersonic vehicle," IEEE/ASME Transactions on Mechatronics, vol. 21, no. 3, pp. 1680-1691, 2016.

[39] X. Bu, X. Wu, M. Tian, J. Huang, R. Zhang, and Z. Ma, "Highorder tracking differentiator based adaptive neural control of a flexible air-breathing hypersonic vehicle subject to actuators constraints," ISA Transactions, vol. 58, pp. 237-247, 2015.

[40] Y. Yuan, Z. Wang, L. Guo, and H. Liu, "Barrier Lyapunov functions-based adaptive fault tolerant control for flexible hypersonic flight vehicles with full state constraints," IEEE
Transactions on Systems, Man, and Cybernetics: Systems, vol. 50 , no. 9, pp. 1-10, 2020.

[41] S. Mobayen and F. Tchier, "A new LMI-based robust finitetime sliding mode control strategy for a class of uncertain nonlinear systems," Kybernetika-Praha-(KYBERNETIKA), vol. 51, no. 6, pp. 1035-1048, 2015.

[42] S. Mobayen and F. Tchier, "Composite nonlinear feedback control technique for master/slave synchronization of nonlinear systems," Nonlinear Dynamics, vol. 87, pp. 1731-1747, 2017.

[43] S. Mobayen and F. Tchier, "An LMI approach to adaptive robust tracker design for uncertain nonlinear systems with time-delays and input nonlinearities," Nonlinear Dynamics, vol. 85, no. 3, 2016.

[44] I. Nagesh and C. Edwards, "A multivariable super-twisting sliding mode approach," Automatica, vol. 50, no. 3, pp. 984-988, 2014.

[45] A. Pati and R. Negi, "Super-twisting algorithm-based integral sliding mode control with composite nonlinear feedback control for magnetic levitation system," International Journal of Automation \& Control, vol. 13, no. 6, p. 717, 2019.

[46] J. Cui, F. Liu, A. Zhang, Y. Hou, and G. Wang, "Adaptive robust control of locomotive brake cylinder with sto," Electronic Design Engineering, vol. 28, no. 7, 2020.

[47] H. Alwi and C. Edwards, "An adaptive sliding mode differentiator for actuator oscillatory failure case reconstruction," Automatica, vol. 49, no. 2, pp. 642-651, 2013. 